\title{
Development and Optimization of an Electrospraying Device for the Continuous Collection of Nano- and Microparticles
}

\author{
Dirk Grafahrend ${ }^{1}$, Philip Jungbecker ${ }^{2}$, Gunnar Seide ${ }^{2}$, Holger Leonards ${ }^{1}$, Thomas Gries ${ }^{2}$, \\ Martin Möller ${ }^{1}$ and Doris Klee ${ }^{*}, 1$ \\ ${ }^{I}$ DWI e.V. and Institute of Technical and Macromolecular Chemistry, RWTH Aachen, Pauwelsstr. 8, D-52056 Aachen, \\ Germany \\ ${ }^{2}$ Institut für Textiltechnik der RWTH Aachen, Eilfschornsteinstraße 18, D-52056 Aachen, Germany
}

\begin{abstract}
The development, fabrication and testing of a compact electrospray device are presented with the dual goal of increasing yields and total particle amounts by orders of magnitude and retaining the monodispersity of the generated polymeric particles. A new and optimized electrospraying device producing $285 \mathrm{mg} / 24 \mathrm{~h}$ monodisperse micro-sized particles with a yield of $79.2 \%$ was developed and tested. The new design allowed continuous particle production and collection. By optimization of the chamber design, high velocities in combination with reduced turbulences led to increasing yields. In comparison to a simple electrospraying configuration, the new device enabled collection 14.25 times more particles in $24 \mathrm{~h}$. Besides this significant increase in particle amounts and numbers, collection effectiveness increased as well. While a basic electrospraying device allowed collection of only $34.7 \%$ of the generated particles, the yields determined for the new device were $79.2 \%$. We report a 2.28 times increase in effectiveness combined with the potential to generate continuously particles with enhanced productivity while maintaining monodispersity of the particles. This modified electrospraying device may facilitate the production of micro- and nano-sized particles for biomedical and pharmaceutical applications in relevant amounts and reduced losses inside the device.
\end{abstract}

\section{INTRODUCTION}

The incorporation of drug into a biodegradable polymer matrix to prepare drug-loaded polymer micro/-nanospheres has been of interest primarily in creating drug delivery vehicles. Drug loaded monodisperse microparticles with sizes (aerodynamic radius) ranging from 1 to $5 \mu \mathrm{m}$ proved suitable for pulmonary delivery and have tremendous potential for the treatment of several chronic diseases including asthma. Novel microparticle formulations as drug delivery systems showing controlled and retarded release have been developed. Degradable and biocompatible microparticles with entrapped drugs are of great interest as well as the development of simple and cost-effective techniques to produce them. Especially aliphatic polyesters with hydrolysable backbones are promising candidates to control the release of drugs entrapped into microparticulate systems. Among them, one of the most commonly employed systems is microparticles fabricated from poly(D, L-lactideco-glycolic acid) (PLGA). These copolymers are attractive for this application due to many favorable characteristics such as good biocompatibility, their ability to degrade into natural metabolites and their safety profile for human use [1]. Electrospraying, or electrohydrodynamic atomization, is a process that relies on a high electrostatic force to break liquid into fine charged droplets. Electrospraying has been applied to many fields, ranging from the generation of

*Address correspondence to this author at the Institute of Technical and Macromolecular Chemistry, RWTH Aachen Pauwelsstr. 8, D-52056 Aachen, Germany; Tel: +49-(0)2418023335;

E-mail:klee@dwi.rwth-aachen.de monodisperse spray of ultra-fine drops to analysis of proteins by mass spectroscopy as well as industrial varnishing processes (paint spraying). More recently, the exploration of electrospraying to manufacture micro- and nanoparticles for drug delivery is becoming a new focus of attention [2]. With this technique different structural drug loaded microparticles, e.g. matrix-drug microparticles, drug-encapsulated core-shell particles, can be produced. At the same time this technique also presents some interesting advantages. For example, Xie et al. have electrosprayed paclitaxel/PLGA microparticles [3], whose diameter can be well controlled using the scaling laws of electrospraying [4]; Ijsebaert et al. have firstly demonstrated that the aerosol microparticles with right diameters are potentially useful as inhalation devices of pulmonary drug delivery, which can be achieved using a modified electrospraying technique [5]. Still, this technique in the area of preparing the drug-loaded particles has not been fully developed. Since electrospraying was first systematically studied by Zeleny [6, 7] and theoretical explained by Taylor [8], many researchers have investigated the mechanisms that induce an electric charge on the surface of a liquid and stretch the meniscus in the direction of the electric field. The physical process when the liquid meniscus is subjected to a strong electric field and electrostatic forces elongate the liquid to form a cone-jet, has been studied and reported by several groups and can be found elsewhere [912]. Briefly, in DC electrospraying charge separation takes place in the liquid meniscus upon application of an electric field between the tip of a capillary from which the liquid meniscus emanates and a grounded electrode placed in a distance. Tangential ionconduction then occurs along the electric double layer formed at the interface thus resulting in 
co-ion accumulation and hence a singular electric field at the meniscus tip. The repulsion between the co-ions at the meniscus tip then results in Coulombic fission wherein a thin liquid jet emanates from the tip once the repulsive force exceeds the surface tension. The co-ion accumulation at the meniscus tip is also the reason why the drops produced as a result of various instabilities suffered by the jet carry a net charge [13].

Several electrospraying modes have been observed [10], the predominant mode being the cone-jet mode in which liquids meniscus assumes the shape of a sharp cone, obtained by balancing the electrostatic and capillary pressure acting on a conical surface for ideal, static equilibrium conditions [8]. For a perfectly conducting liquid, Taylor [8] showed by considering the static equilibrium balance between the capillary and Maxwell stresses that a conical meniscus with a half angle of $49.3^{\circ}$ is produced.

From the previous description of theory, it is clear that the electrospraying process can be manipulated by a number of variables. Doshi and Reneker classified the parameters that control an electrostatic process like electrospraying in terms of solution parameters, process parameters, and ambient parameters [14]. Solution parameters include the viscosity, conductivity, surface tension, polymer molecular weight, dipole moment, and dielectric constant. The effects of the solution properties can be difficult to isolate since varying one parameter can generally affect other solution properties. Process parameters include the flow rate, electric field strength, distance between tip and collector, needle tip design, and collector composition and geometry. Ambient parameters include temperature, humidity, and air velocity [15]. For detailed discussion on the effects of the most influential of these parameters on electrospraying/electrospinning of PLGA solutions, we refere to the literature by Berkland et al. [16]. Besides the previously described simple electrospraying configuration several more elaborated devices have been developed. A special nozzle system allowing control over the surface nano-structure has been reported by Berkland et al. [16]. A thin sharpened tungsten electrode inside a glass capillary is responsible for increased process control in the flow-limited field-injection electrostatic spraying (FFESS) configuration. Another nozzle modification especially for micro- and nano-encapsulation is the use of coaxial electrospraying configurations [17]. A coaxial electrospraying nozzle basically consists of a larger outer capillary through which a polymer solution can be fed and a smaller inner capillary through which the material to be encapsulated is supplied. If both liquids have appropriate physical properties (viscosity, conductivity etc.) and are immiscible core-shell particles can be obtained. Recently, Yeo et al. [18] described an electrospraying configuration using high-frequency AC electric fields above $10 \mathrm{kHz}$. They report on the potential of this method for scalability to both portable in-situ delivery devices and mass production lines. Applications are focused on two major areas, namely, micro/nano-encapsulation for drug delivery and on the other hand fiber synthesis for tissue/orthopedic engineering and wound healing. Several different electrode designs and collection chambers have been developed and proposed over the years. For example, the use of a ring-electrode for the production of cholesterol microparticles has been reported by Reyderman et al. [19]. In this case, the ring-electrode was positioned directly on the opening of the collection tube. More recently, an electrospraying configuration sometimes referred to as "Delft-type" since it was constructed for the first time in the Technical University of Delft (Netherlands) has been reported [20]. The set-up consists of a cylindrical glass tube with tapered ends. One end acts as an inlet for air and the other end ducts the produced particles via a heater to the collection side. The spraying nozzle is positioned in a glass side tube in which also the counter electrode ring is placed close to the main cylinder. The distance between the nozzle-tip and the ring is $10 \mathrm{~mm}$. The discharge needle is inserted in the glass cylinder opposite to the spraying nozzle. The airflow through the system equals $30 \mathrm{~L} / \mathrm{min}$. Particles are collected on a membrane filter placed downstream of the set-up [21]. The two major advantages of this electrospraying configuration are continuous collection of particles and neutralization of the charges on generated droplets and resulting powder formulations. In order to increase and gain better understanding of the potential of electrospraying for encapsulation, the objective of this study was to optimize the electrospraying device in a step-wise manner. Starting from a simple configuration, we modified the chamber design and introduced a cyclone-type particle collector. The main objective was to develop a device suitable for continuous particle collection, higher yields and collection of isolated particles, and non-aggregated powder formulations for medical and pharmaceutical applications.

\section{EXPERIMENTAL PART}

\section{Materials and Methods}

Acetone was purchased from Sigma-Aldrich $\mathrm{GmbH} \&$ Co. KG. (Taufkirchen, Germany). Resomer RG502H (provided by Boehringer Ingelheim, Germany) is a PLGA having a molecular weight $\left(\mathrm{M}_{\mathrm{n}}\right)$ of $12.2 \mathrm{kDa}$ and consists of 50:50 mixture of lactic acid: glycolic acid and an inherent viscosity between $0.16-0.24 \mathrm{dl} / \mathrm{g}$. This polymer was uncapped, meaning that it has free carboxylic acid groups. The electrospraying devices consisted of the following three main parts: An Eltex KNH35 (Germany) high voltage generator was utilized to charge the solution, a cyclone type particle collector (Respirable Dust Aluminum Cyclone, SKC Inc., USA) and a vacuum pump (Vakuumbrand, Germany). All other parts were manufactured at the DWI workshop and tailored for each device. In all three electrospraying configurations, a HA 11 plus (Harvard Apparatus, purchased from Hugo-Sachs Elektronik GmbH, March-Hugstetten, Germany) was used to pass polymer solutions through the nozzles at controlled flow rates.

\section{Electrospraying Experiments with the Simple Configuration (Device I)}

The basic electrospraying configuration consisting of a syringe through which a polymer solution was pumped at a constant rate and which was connected to a high voltage power supply and a grounded target for collection of the particulate is shown in Fig. (1).

Target and spraying nozzle were enclosed in a $450 \mathrm{X} 450$ X $800 \mathrm{~mm}$ poly(methyl 2-methylpropenoate) (PMMA) box to eliminate the possibility of any contamination of the environment. After electrospraying and disconnecting the high voltage supply, the box was opened and the sample 
removed. In the simplest electrospraying experiments, a polymer solution (1-3 wt\% PLGA in acetone) was supplied to the spraying nozzle at a constant rate of $0.08 \mathrm{~mL} / \mathrm{h}$. The distance between the spraying nozzle and the grounded collector was $200 \mathrm{~mm}$ and an applied voltage of $25 \mathrm{kV}$ proved to be optimal for production of monodisperse spherical polymer particles in nano- to micrometer scale. Particles were collected for $20 \mathrm{~min}$ and the collector was exchanged by a new one. Particles collected over a period of $24 \mathrm{~h}$ were transferred into $1.5 \mathrm{~mL}$ Eppendorf tubes and the yield was determined. Average particles yields are presented, which result from three independent production cycles.

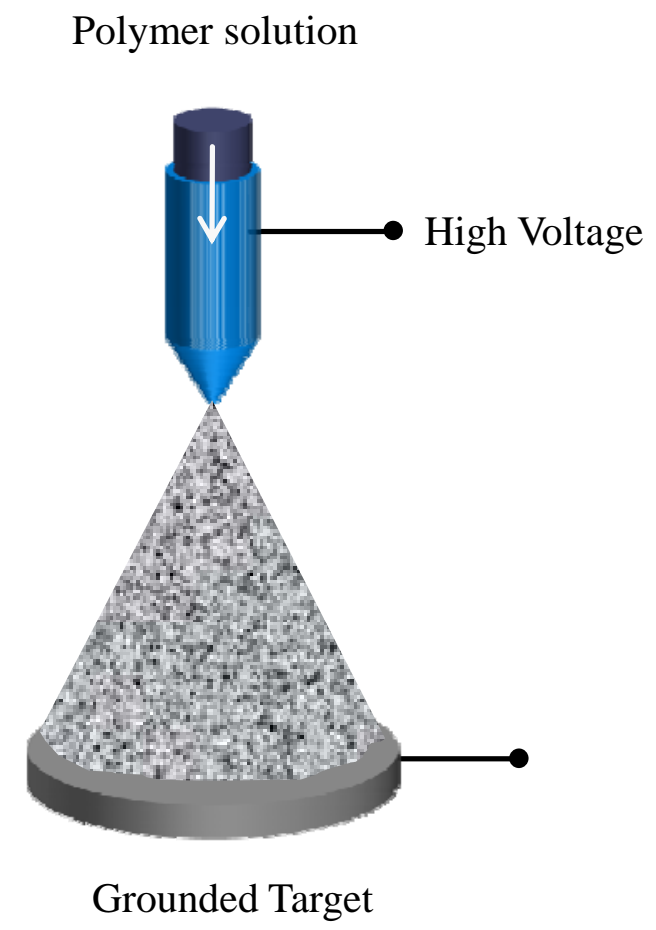

Fig. (1). Basic electrospraying configuration consisting of a syringe through which a polymer/drug solution was pumped at a constant rate and which was connected to a high voltage power supply, and a grounded target for collection of the particles.

\section{Electrospraying Experiments with a Configuration for Continuous Collection (Device II)}

In addition to the three main elements of the basic configuration, this set-up consisted of a spraying chamber and a cyclone-type particle collector (see Fig. 2).

The experimental basically consisted of a syringe filled with a polymer solution and a grounded electrode encapsulated in commercial PMMA tubing. A PMMA tubing with a length of $680 \mathrm{~mm}$ and a diameter of $170 \mathrm{~mm}$ was used with adequate closure heads for this purpose. A round air inlet of $30 \mathrm{~mm}$ was concentrically recessed in one of the two closure heads, while a Teflon Tubing with an outer diameter of $10 \mathrm{~mm}$ (inner diameter $8 \mathrm{~mm}$ ) was fixed at the second closure head. The length of this Teflon tubing connecting the spraying chamber and the Respirable Dust Cyclone (SKC Inc, USA) was $300 \mathrm{~mm}$. Both, nozzle and ground electrode were positioned $200 \mathrm{~mm}$ from the air inlet opposite to each other. The distance between the tip of the electrospraying nozzle and the ground electrode was fixed at
$120 \mathrm{~mm}$. A constant air-flow of $28 \mathrm{~L} / \mathrm{min}$ through the device and cyclone was applied with a vacuum pump (Vakuumbrand, Germany). A polymer solution (1 wt \% PLGA in acetone) was fed to the spraying nozzle at a constant rate of $1.5 \mathrm{~mL} / \mathrm{h}$. Particles were collected in cyclone-type particle collector. Particles were produced over a period of $24 \mathrm{~h}$ and collected continuously on a filter membrane $(0.2 \mu \mathrm{m}$ cellulose filter membrane, Millipore, Germany). Afterwards the particles were transferred into 1.5 $\mathrm{mL}$ Eppendorf tubes and the yields determined. Average particles yields are presented, which result from three independent production cycles.

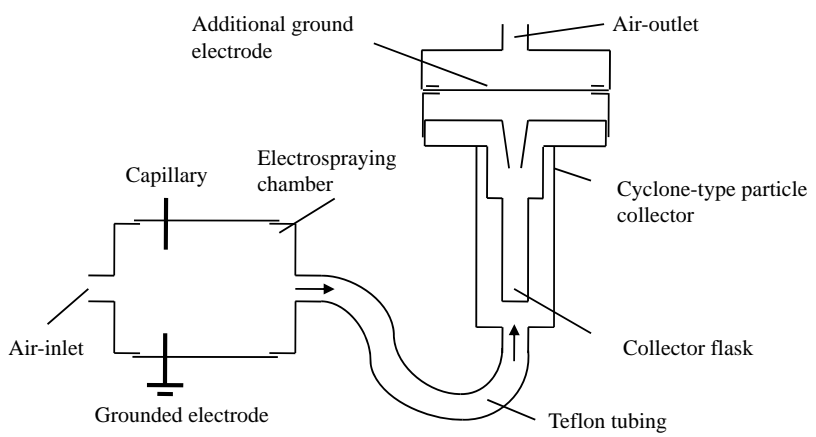

Fig. (2). Electrospinning configuration for continuous particle production and collection. In addition to the elements of the basic configuration, this set-up consisted of a spraying chamber and a cyclone-type particle collector.

\section{Electrospraying Experiments with the Optimized Configuration for Continuous Collection (Device III)}

In contrast to the previously described electrospraying configurations, this device consisted of a glass chamber with optimized geometry and design. All described measures except the spraying chamber have been retained unchanged. A glass separation funnel was modified with two glass tubings (length: $60 \mathrm{~mm}$ ) with ground neck NS 29. As shown in Fig. (3), the tubings were fixed to the separation funnel at the place of maximum diameter in opposing positions.

As precursor for the production of particles, a solution of polymer was supplied to the spraying nozzle. PLGA was dissolved in dry acetone to make a $1 \mathrm{wt} \%$ solution. The flow rate of the solution was usually in the range of $1.5 \mathrm{~mL} / \mathrm{h}$. High voltage power supply set on $20-25 \mathrm{kV}$ was connected to the nozzle while the second electrode positioned opposite to this one remained on ground potential. The applied voltage was adapted to a current of $0.3 \mu \mathrm{A}$ through the device. Particles were collected on a filter membrane $(0.2$ $\mu \mathrm{m}$ cellulose filter membrane, Millipore, Germany) inside the cyclone type particle collector (Respirable Dust Aluminum Cyclone, SKC Inc, USA) downstream of the setup. All presented particles on the scanning electrone microscope images (SEM-images) (see Fig. 4) were generated in the set-up shown in Fig. (3). Average particles yields are presented, which result from three independent production cycles.

\section{Air-Flow Simulations and Calculation of Isolated Particle Tracks}

All simulations have been computed by the Institut für Textiltechnik of RWTH Aachen University (Eilfschornstein- 
straße 18, D-52062 Aachen, Germany) and were kindly provided for comparison with the experimental results. The finite volume method was used for simulation of the air-flow and the corresponding velocities. As precondition for the simulation of droplet/particle tracks after injection into the air-flow we assumed every particle hitting the wall as loss. This assumption seemed realistic as every droplet or particle in a non-dry state tends to stick to the wall due to the adhesiveness and viscousness of the material swollen in acetone. In addition the non-neutralized charges of the particles attributed to the stickiness as well. Only dry particles could detach from the walls and reach the place of collection. Although the Reynolds number was about 250 in the tube of device II, a turbulence model (k-e) was used as the flow was not developed. Therefore, some turbulent areas, which have also been observed, could be simulated.

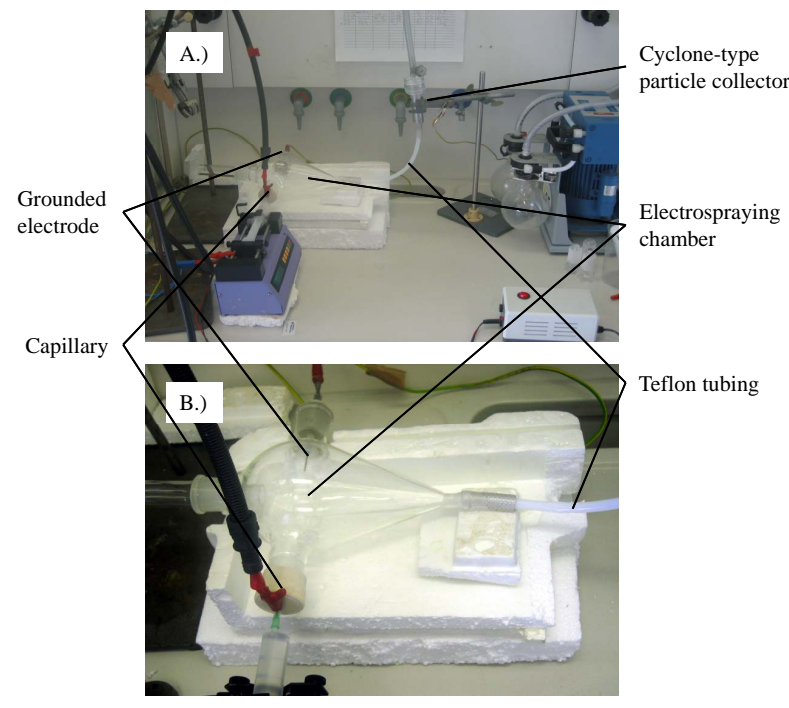

Fig. (3). Images of the electrospraying device. A) Overview of the electrospraying configuration consisting of a chamber, syringe with syringe pump, high voltage connections, vacuum pump, tubing and a cyclone-type particle collector. B) Detailed view on the electrospraying chamber showing the syringe connected to the high voltage, the grounded electrode, the air-inlet and the connection to the particle collector.

\section{RESULTS}

Electrosprayed biodegaradble polymer particles were generated using three different devices (device I, II, and III). The common parameters used for the production of all particles are given in Table $\mathbf{1}$.

Exemplary, the polymeric system used in this study was PLGA dissolved in acetone to make concentrations of 3 wt $\%$, respectively $1 \mathrm{wt} \%$. The polymer used in this study was kept identical independent of the device used for better comparison. A voltage of $25 \mathrm{kV}$ and needle diameter of 24 ga was used. All three parameters, concentration, flow rate, and nozzle to collector distance had to be adapted to the devices in order to obtain monodisperse particles in the inhalable size range. The optimum conditions for the particle generation were determined for each configuration and device used. Fig. (4) shows SEM images of electrosprayed PLGA particles produced in this study.

Table 1. General Electrospraying Conditions Used for the Generation of PLGA Particles

\begin{tabular}{|c|c|}
\hline Polymer & PLGA \\
\hline \hline Solvent & Acetone \\
\hline Concentration & $1-3 \mathrm{wt} \%$ \\
\hline Distance (nozzle/ground electrode) & $120-200 \mathrm{~mm}$ \\
\hline Voltage & $25 \mathrm{kV}$ \\
\hline Flow rate & $0.08-1.5 \mathrm{~mL} / \mathrm{h}$ \\
\hline Capillary diameter & $24 \mathrm{ga}=1.44 \mathrm{~mm}$ \\
\hline
\end{tabular}

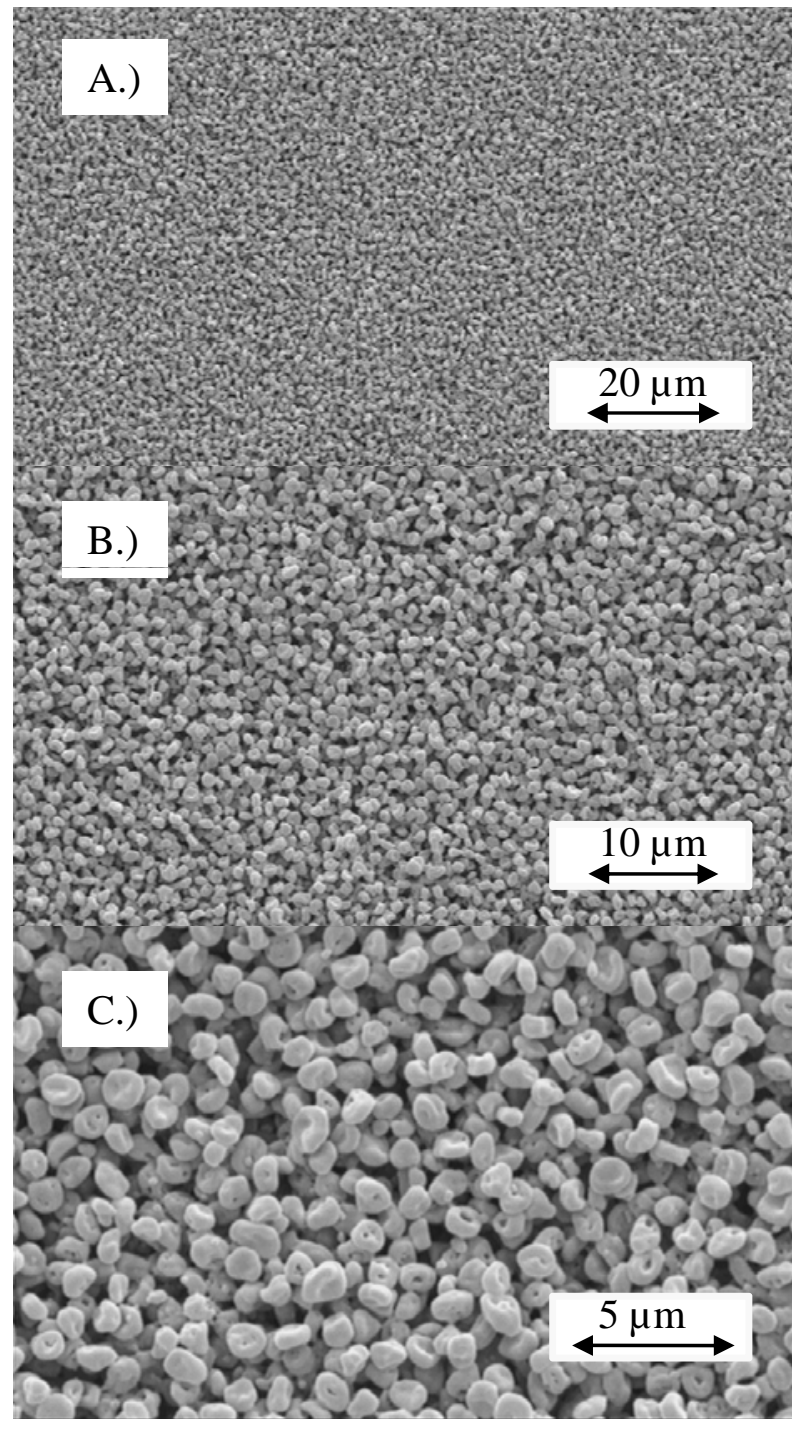

Fig. (4). Electrosprayed PLG particles. A) Magnification $X=1000$, B) magnification $X=2000$, and $\mathbf{C}$ ) magnification $X=5000$.

Different magnifications are shown to illustrate the homogeneity and monodispersity of the generated particles. All particles show spherical shape and smooth surface 
morphologies. The most simple electrospraying configuration (Device I) is schematically shown in Fig. (1). The advantage of such a simple configuration, being easily adjustable distances has to be highlighted. For determination of spray ability and in order to obtain a first set of processing parameter experiments with such a configuration are necessary. The electrospraying parameters for all three devices are summarized in Table 2.

Table 2. Apparatus Specific Electrospraying Conditions (for All Three Devices)

\begin{tabular}{|c|c|c|c|}
\hline Electrospraying Device & I & II & III \\
\hline \hline Concentration [wt\%] & 3 & 1 & 1 \\
\hline $\begin{array}{c}\text { Distance } \\
\text { (nozzle/ground electrode ) } \\
{[\mathrm{mm}]}\end{array}$ & 20 & 12 & 12 \\
\hline Flow rate [mL/h] & 0.08 & 1.5 & 1.5 \\
\hline Air flow [L/min] & - & 28 & 28 \\
\hline Time $[\mathrm{h}]$ & 24 & 24 & 24 \\
\hline Collection & 72 samples & continuous & continuous \\
\hline Yield [mg] & $20 \pm 16$ & $200 \pm 40$ & $285 \pm 22$ \\
\hline
\end{tabular}

A nozzle to collector distance of $120 \mathrm{~mm}$ and a flow rate of $0.8 \mathrm{~mL} / \mathrm{h}$ proved to be best suitable for the production of homogeneous sprays usig device I. As alumina SEM-stubs were used for collection and the surface area of such a collector is very limited, they had be exchanged approximately every $20 \mathrm{~min}$. This was a clear disadvantage as for the production of $20 \mathrm{mg}$ over a period of $24 \mathrm{~h}$ the collector had to be exchanged 72 times. Besides being unpractical and time consuming, collection on 72 different collectors led to higher material losses when the particles are merged to one batch in an Eppendorf tube. During a $24 \mathrm{~h}$ time period, $1.92 \mathrm{~mL}$ corresponding to $57.6 \mathrm{mg}$ polymer were electrosprayed. As only $20 \mathrm{mg}$ were collected, $34.7 \%$ of the theoretical yields were obtained. The rest of the particles was distributed in the whole PMMA-box and could not be located nor collected. In the next electrospraying device (device II) several set-up changes were introduced. Firstly the place of collection and the other electrode were separated. Secondly, a more efficient collector was introduced. Thirdly, the chamber size was decreased in order to minimize surface for particle deposition. Fig. (2) shows an electrospraying device II suitable for continuous particle collection. In order to separate the place of collection and the grounded electrode, we introduced a second capillary connected to ground potential and applied constant air-flow of $28 \mathrm{~L} / \mathrm{min}$ to transport the particles to the collection place downstream. As more sufficient collector a respirable aluminum dust cyclone commonly used for environmental sampling and analysis was introduced. Dependent on the airflow and particle size, particles were either sedimented in a collection flask or collected on a membrane with $0.2 \mu \mathrm{m}$ pores size. In order to decrease the inner surface of the electrospraying device, a tube with an inner diameter of 170 $\mathrm{mm}$ and a length of $680 \mathrm{~mm}$ was used. Best results with this device (device II) were achieved using concentrations of 1 $\mathrm{wt} \%$, a nozzle to target distance of $120 \mathrm{~mm}$, and solution flow rates of $1.5 \mathrm{~mL} / \mathrm{h}$. Total particle amounts of $200 \mathrm{mg}$ were achieved over a period of $24 \mathrm{~h}$. The device specific process parameters are given in Table 2. Theoretical $36 \mathrm{~mL}$ of the polymer solution corresponding to $360 \mathrm{mg}$ of polymer have been generated over a period of $24 \mathrm{~h}$. As only $200 \mathrm{mg}$ $(55.56 \%)$ were collected, $44.44 \%$ of the particles were not located and contribute to losses. In order to identify the reason for these losses, simulations of the air flow inside device II were performed. We underline the qualitative nature of these calculations since the electric field and interactions between charges were not considered.

The program used for the Computational Fluid Dynamics (CFD) inside the chamber was Fluent from ANSYS Germany $\mathrm{GmbH}$, Darmstadt. The simulation was done in three steps: Preprocessing, processing and postprocessing. In the preprocessing phase the chamber geometry with the Teflon tubing was created as a three dimensional CAD drawing. The simulated flow volume ranged from the airinlet of the chamber to the first part of the teflon tubiung. The cyclone-type particle collector was not considered in the simulation since it does not influence the air-flow in the chamber. The geometry is meshed with 335,000 hexahedral cells. Also in the preprocessing phase the boundary conditions were evaluated. We assumed the flow to be turbulent despite of the low volume flow. Taking the given volume flow into account for calculation of the Reynolds' number for the air flow in the spraying chamber and the Teflon tubing, values of 250 and 5000 were determined. Even though the Reynolds number determined for the spraying chamber was lower than the critical value of 2300 , the assumption of a turbulent flow was justified due to the turbulences induced by the sharp edges at the air-inlet. Inside the spraying chamber these turbulences continued as a relaminization did not occur in the short distance. As a turbulence model the standard $\mathrm{k}-\varepsilon$ model was used. This model was based on the Reynolds Averaged Navier Stokes Equations (RANS), which means that eddies were simulated as an average effect of time. In the two-equation model a linear eddy-viscosity was added corresponding to the turbulence. The dynamic viscosity of air was set to $1.72 * 10^{-5}$ $\mathrm{Pa}^{*}$ s, its density to $1.225 \mathrm{~kg} / \mathrm{m}^{3}$. The flow was modeled as incompressible and adiabatic. In the processing phase the discretized conservation equations were solved iteratively starting from an initial solution. When convergence was reached the flow field has been studied during post processing. The simulated air-flow inside the spraying chamber and the tubing and corresponding velocity vectors are shown in Fig. (5).

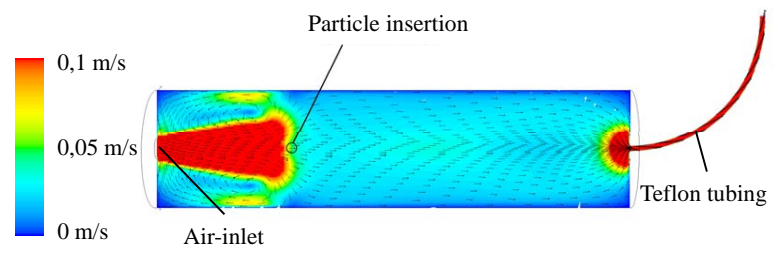

Fig. (5). Simulation of the air-flow and local velocities in the electrospraying chamber connected to a vacuum pump transporting the air at a rate of $28 \mathrm{~L} / \mathrm{min}$. The place where the needle and the spray enter the chamber is marked with circle. 
At the inlet the air was transported with velocities of approximately $0.1 \mathrm{~m} / \mathrm{s}$ into the spraying chamber. The entering air-stream expanded until it collapsed after $200 \mathrm{~mm}$. It partially flowed back near the chamber walls so that a recirculation area builded up. In the rest of the spraying chamber very low velocities of $0.03 \mathrm{~m} / \mathrm{s}$ were calculated. The air was transported with approximately $9.4 \mathrm{~m} / \mathrm{s}$ through the tubing to the respirable aluminum cyclone. As we expected the turbulent air flow to be the main reason for the particle losses, we examined the inside of the spraying chamber and the deposition pattern after $24 \mathrm{~h}$ of continuous electrospraying. When opening the tubular spraying chamber, it was visible that some areas are covered with a thin whitish layer. The deposition pattern was inhomogeneous and showed areas of increased deposition and almost no deposition. While the surface around the position were the spraying nozzle and the target are introduced in the tube $( \pm 50 \mathrm{~mm})$ was covered with a larger number of particles (visible by the white color), the inner surface between the air-inlet and this position did show almost no particles. The area towards the Teflon tubing connecting the spraying chamber and the cyclone was covered with a thinner whitish layer. The closure head on the collection side itself and especially the area around the outlet showed most deposited particles. In a next step the particle flow inside the spraying device with the previously described air-flow was calculated using Fluent again. The sphere particles started from a virtual line between capillary and the gronded target. They were given a specific density of $0.7 \mathrm{~g} / \mathrm{cm}^{3}$ and a diameter distribution between 1 and $10 \mu \mathrm{m}$ with an average of $5 \mu \mathrm{m}$. These particles started with no initial velocity and are transported by aerodynamic forces. As the volume fraction of the particles in the air-flow was less than $1 \%$ an interaction between air-flow and particle motion was not considered. A discrete random walk model which is implemented in Fluent was used for the stochastic tracking of the particles. The model causes a turbulent dispersion of the particles which led to an arbitrary number of particle tracks. Fig. (6) illustrates some of the simulated particle tracks inside the electrospraying chamber.

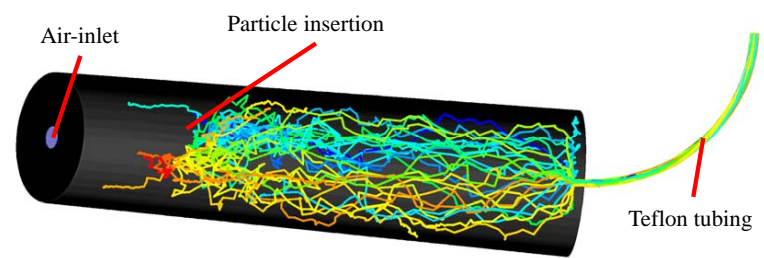

Fig. (6). Simulated particle tracks in the electrospraying chamber. Every particle hitting the wall is expected to stick to the wall. These particles attribute to losses and lower yields. Approximately half of the generated particles hit the wall.

Electrostatic interaction between the particles and with the surface of the electrospraying chamber have been ignored in the simulation. The differences in the particle motion can be explained by the turbulences. From the calculations, provided that every particle hitting the wall does not reach the collection side, we concluded that approximately half of the generated particles reached the collector. Fig. (7) shows the distribution of the particles on the wall.

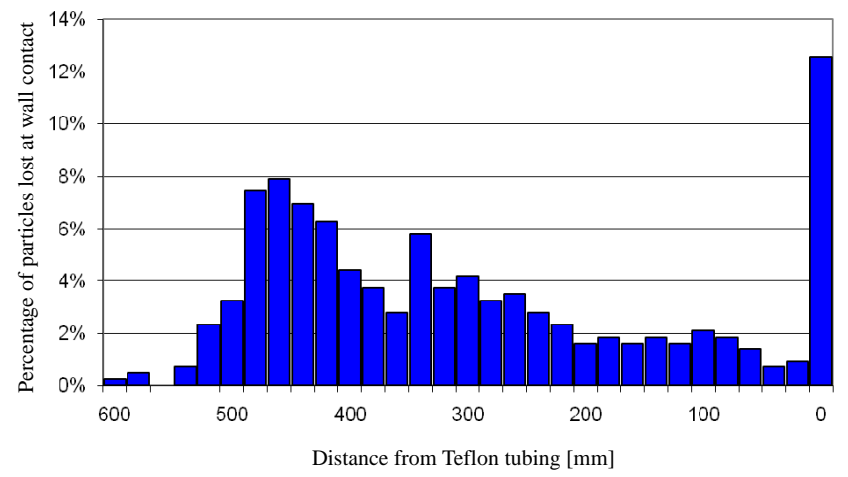

Fig. (7). Simulated Particle distribution inside the electrospraying chamber. $12 \%$ of the produced particles hit the wall at the wall on the collection side where the tube to the cyclone-type particle collector is located (indicated with 0 ). Another place where a high particle concentration is predicted is in the area of the entering needle (400 to $500 \mathrm{~mm}$ ).

The distances on the center line represent the distances from the out-let and the Teflon tube connecting the spraying chamber and the cyclone. The simulation results show particles distributed almost over the whole inner surface. A higher particle concentration has been found in the areas where the particles were produced, and where the back flow was calculated as well as on the head closure with the outlet. Approximately $12 \%$ of the generated particles were deposited around the out-let and the Teflon tubing. In order to minimize particle losses and to increase the yields turbulences inside the spraying chamber had to be decreased. Generally, avoiding sharp edges as well as corners could have been able to diminish these turbulences. Fig. (8) shows a schematically drawn design of a spraying chamber with optimized geometry for minimal turbulences.

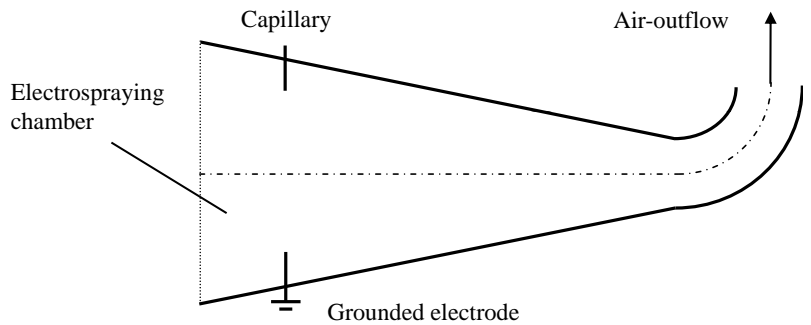

Fig. (8). A new chamber geometry with reduced air currents and reduced local air-velocity.

Especially the funnel like shape of the spraying chamber led to a homogeneous velocity distribution with most velocity vectors directed towards the collector side. One clear disadvantage of this modified geometry was the lower velocities in the area of particle generation. Just by moving the spraying nozzle (in device II) $50 \mathrm{~mm}$ in the direction of the collector, particle yields droped down dramatically (from $200 \pm 40 \mathrm{mg} / 24 \mathrm{~h}$ to approximately $40 \mathrm{mg} / 24 \mathrm{~h}$ ). In this position of electrospraying device II velocities of approximately $0.03 \mathrm{~m} / \mathrm{s}$ were insufficient to deflect the particles from their trajectories towards the grounded electrode and carry them to the collector downstream of the device. Combination of higher velocities and less turbulence were combined by designing a smaller chamber with less dead volume. The schematic drawing in Fig. (9) reflects on possible new design. 


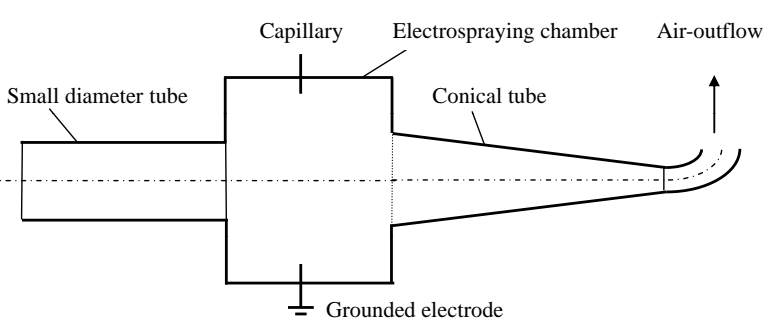

Fig. (9). Schematic drawing of a new chamber design for optimized air-flow. Due to the reduced inner volume, higher local airvelocities are expected. Additionally, due to the conical shape reduced currents should be obtained.

The funnel-like shape of the spraying area of the chamber, the tube like air-inlet and the two added tubings for the two capillaries (spraying nozzle and grounded electrode) combined higher air-velocities and less turbulence, respectively current. With an upfront engineering approach the flow field in different designs was simulated until the optimum proportions have been found. The calculated contours of velocity inside the optimum spraying chamber and corresponding velocity vectors are shown in Fig. (10).

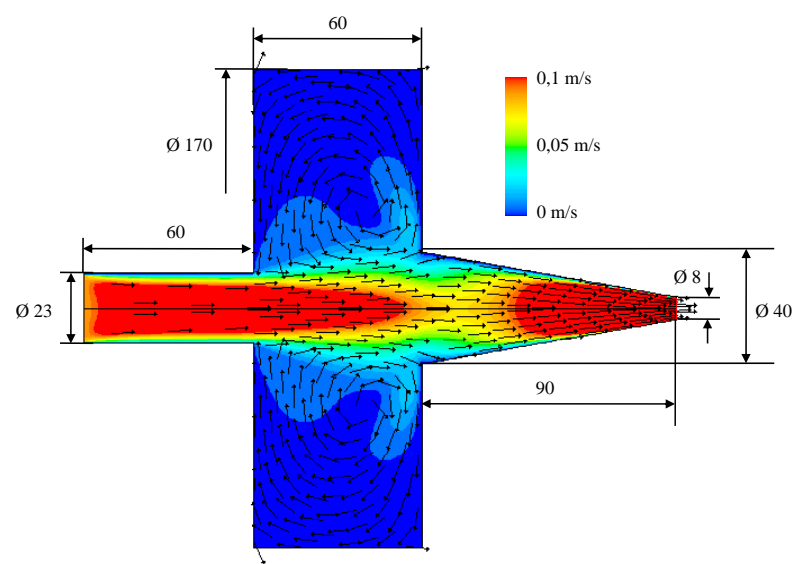

Fig. (10). Simulation of the air-flow in the optimized chamber geometry. Higher local air-velocities and fewer currents in the center region of the electrospraying chamber were predicted.

Fig. (3) is an image of the optimized electrospraying configuration (device III). The spraying chamber had a length of $250 \mathrm{~mm}$ and was made of glass. The overview image of the set-up illustrates how all parts were connected while Fig. (3B) gives a detailed impression of the spraying chamber. The shown device was used for the generation of PLGA particles. The highest flow rate at which a stable monodisperse electrospray has been sustained was again 1.5 $\mathrm{mL} / \mathrm{h}$, which corresponded to a production rate of 360 $\mathrm{mg} / 24 \mathrm{~h}$. At larger flow rates, on the order of $2 \mathrm{ml} / \mathrm{h}$ it was still possible to stabilize the conical meniscus but monodispersity was compromised. Still higher flow rates resulted in unstable behavior of the conical meniscus and were not examined. $285 \mathrm{mg}$ particles were collected during a $24 \mathrm{~h}$ period, which corresponds to a yield of $79.2 \%$.

\section{DISCUSSION}

To obtain particles of narrow size distribution, voltage and liquid flow rate were fixed in such a way that the system operates in a stable mode. The different operation modes have been reported in literature. Especially the cone-jet mode has been attributed to a stable process [22-25], which is necessary for the generation of monodisperse particles (and droplets). One method of cone stability control is monitoring the voltage of the electric current flowing through the nozzle. When electrospraying operates in the cone-jet mode, the electric current is stable, usually in the range of one to tens of microampere [21]. For the production of certain particle sizes, a final tuning of the system by changing the liquid flow or voltage is necessary as the particle sizes varied with the device used. While morphology and internal structure of particles produced are known to depend strongly on the precursor type and solvent properties [17], yields and aggregation depended on the device used. The results of the electrospraying experiments carried out using device I were promising in terms of particle homogeneity, size and reproducibility. The first device (device I) was kept as simple and flexible as possible. Therefore it consisted only of the main components, namely: high voltage power supply, syringe pump, a grounded target (aluminium SEM-stub) and a spraying chamber (in this case a large PMMA box in which the target and syringe are introduced). This device allowed a first feasibility check and determination of processing pararmeters. Yet we faced problems in obtaining significant particle amounts as well as aggregation and agglomeration of these particles. Especially these three disadvantages limited the applicability of this technique for particle generation in the field of drug delivery systems (for example pulmonary drug delivery). For biomedical and pharmaceutical applications such yields and total particle amount were not sufficient and a scale-up was needed. In order to be able to collect particles continuously over longer time periods and to increase yields and productivity, the electrospraying configuration was modified. For this reason several modifications and changes had to be introduced. In this second device the particles were generated at the spraying nozzle, attracted by the target (grounded electrode) and carried to the cyclone-type particle collector placed downstream by the air-flow. This modified device allowed continuous collection of non-agglomerated particles. Especially the continuous air-flow through the device led to sufficient solvent evaporation and removal and therefore less aggregation. The optimum conditions for the generation of spherical solid particles proved to be altered significantly from the one described previously for device I. While the distance between the spraying nozzle and the grounded target was reduced $40 \%$, flow rate has been increased 20 fold. Even if at the same time the sloution concentration was decreased to $1 \mathrm{wt} \%$, some clear benefits of this new device (device II) were identified. In comparison to device I continuous particle collection, prevention of aggregation and agglomeration combined with higher yields were some of these benefits of the second device. In comparison to the particle amounts produced with device I this was a ten times increase of collected particles. Besides an improved comfort, particle amounts in the gram scale have been produced within a few days. In comparison to the previously described results this first modification already meant a significant increase. As mentioned previously, still approximately $45 \%$ of the electrosprayed particles were not transferred to the collection side (device II). The turbulences induced by the sharp edges at the air-inlet together with the low velocities 
caused a high turbulence intensity, which meant that the velocity fluctuation was in the same size as the average velocity. The air was pumped out of the spraying chamber at the outlet through the Teflon tubing. The simulation of the particle trajectories inside the second device illustrated these losses. Half of the generated particles hit the wall of the spraying chamber during their flight. The calculations, provided that every particle hitting the wall did not reach the collection side, showed that approximately half of the generated particles reached the collector and were in good agreement with the experimental results. Hence, we assumed that every particle hitting the wall on its flight adhered. Therefore, further increasing productivity by decreasing turbulences and at the same time providing constantly high air velocity inside the device was achieved in device III. Changing the the chamber geometry in the previousley described manner (see reults) decreased turbulences as shown by the simulation. At the inlet the air was transported with velocities of approximately $0.1 \mathrm{~m} / \mathrm{s}$ into the spraying chamber. The velocity of the air flow in the center area of the spraying chamber kept constantly that high. No significant differences between the air inlet and the connection to the Teflon tubing were identified. The velocity of the air in the area of the two capillaries droped down to $0 \mathrm{~m} / \mathrm{s}$. Therefore, the particles generated at the spraying nozzle were firstly attracted by the grounded electrode, entered the area of high air velocity, were distracted and carried towards the collector. The residual solvent evaporated during their flight and solid particles were collected. Optimization of the chamber design clearly increased particle amounts and yields.

\section{CONCLUSION}

A new electrospraying device for the continuous production of increased particle amounts and higher yields of polymeric materials has been developed. This device utilized several improvements over the commonly used electrospraying configurations including continuous collection over long time periods via separation of the grounded electrode and the place of particle collection. Using an aerodynamic transport system for the particles to the cyclone-type particle collector led to the collection of solid, non aggregated and agglomerated sprays. Optimization of the chamber design further increased the obtained yields. Minimization of turbulences in the spraying chamber while maintaining relatively high air velocities of $0.1 \mathrm{~m} / \mathrm{s}$ led to yields of $79.2 \%$ corresponding to $285 \mathrm{mg} / 24 \mathrm{~h}$. By discretely varying chamber and device design, efficiency in terms of particle amounts and yields were realized. Thus relevant amounts for biomedical and pharmaceutical applications can be produced, for example formulations for controlled drugdelivery devices. Used to create specific formulations or coating existing drug particles, electrospraying technology provides a simple and robust technique for fabrication of dry powder formulations consisting of monodisperse particles with precisely define size range and defined nano-structures from a broad range of biocompatible polymeric materials.

\section{ACKNOWLEDGEMENTS}

The authors would like to thank Dr. Michael Krüger and Dr. Regis Cartier for discussions and helpful remarks. Julia Lleixa Calvet and Ralf Krüll are kindly acknowledged for their support. Additionally, the authors wish to thank the BMBF, Bundesministerium für Bildung und Forschung, for financial support of the research project "NanoInhale" (13N8888). Boehringer Ingelheim Pharma GmbH \& Co. KG. are kindly acknowledged for providing chemicals. This work was partially supported by DFG Graduiertenkolleg 1035 "Biointerface".

\section{REFERENCES}

[1] Fernandez-Carballido, A.; Pastoriza, P.; Barcia, E.; Montejo, C.; Negro, S. PLGA/PEG-derivative polymeric matrix for drug delivery system applications: Characterization and cell viability studies. Int. J. Pharm., 2007, 352, 50-57.

[2] Hong, Y.; Li, Y.; Yin, Y.; Li, D.; Zou, G. Electrohydrodynamic atomization of quasi-monodisperse drug-loaded spherical/wrinkled microparticles. J. Aerosol Sci., 2008, 39, 525-536.

[3] Xie, J.; Marijnissen, J. C. M.; Wang, C.-H. Microparticles development by elctrohydrodynamic atomization for the local delivery of anticancer drug to treat C6 glioma in vitro. Biomaterials, 2006, 27, 3321-3332.

[4] Hartman, R. P. A.; Borra, J. P.; Brunner, D. J.; Marijnissen, J. C. M.; Scarlett, B. The evolution of elctrohydrodynamic sprays produced in the cone-jet mode, a physical model. J. Electrostat., 1999, 47, 143-170.

[5] Ijsebaert, J. C.; Geerse, K. B.; Marijnissen, J. C. M.; Lammers, J. W. J.; Zanen, P. Electrohydrodynamic atomization of drug solutions for inhalation purposes. J. Appl. Physiol., 2001, 91, 27352741.

[6] Zeleny, J. The electrical discharge from liquid points and a hydrostatic method of measuring the electric intensity at their surface. Phys. Rev., 1914, 3, 69-91.

[7] Zeleny, J. Instability of electrified liquid surfaces. Phys. Rev., 1917, 10, 1-6.

[8] Taylor, I. G. Disintegration of water drops in an electric field. Proc. R. Soc. Lond. A, 1964, 280, 383-397.

[9] Cloupeau, M.; Prunet-Foch, B. Electrostatic spraying of liquids in cone-jet mode. J. Electrostat, 1989, 22, 135-159.

[10] Cloupeau, M.; Prunet-Foch, B. Electrohydrodynamic spraying functioning modes: A critical review. J. Aerosol Sci., 1994, 25, 1021-1036.

[11] Fernandez, de la Mora J. The effect of charge emission from electrified liquid cones. J. Fluid Mech., 1992, 243, 561-574.

[12] Gomez, A.; Thang, K. Charge and fission of droplets in electrostatic sprays. Phys. Fluids, 1994, 6, 404-414.

[13] Yeo, L. Y.; Chang, H.-C. High frequency AC electrosprays: mechanisms and applications. Adv.Fluid Mech. 2006, VI, 223-231.

[14] Doshi, J.; Reneker, D. H. Electrospinning process and applications of electrospun fibers. J. Electrostat., 1995, 35, 151-160.

[15] Pham, Q. P.; Sharma, U.; Mikos, A. G. Electrospinning of polymeric nanofibers for tissue engineering applications: a review. Tissue Eng., 2006, 12, 1197-1211.

[16] Berkland, C.; Pack, D. W.; Kim, K. (K.) Controlling surface nanostructure using flow-limited field-injection electrostatic spraying (FFESS) of poly(D,L-lactide-co-glycolide). Biomaterials, 2004, 25, 5649-5658.

[17] Loscertales, I. G.; Barrero, A.; Guerro, I.; Cortijo, R.; Marquez, M.; Ganan-Calvo, A. M. Micro/nano encapsulation via electrified coaxial liquid jets. Science, 2002, 295, 1695-1698.

[18] Yeo, L. Y.; Gagnon, Z.; Chang, H.-C. Technical note: AC electrospray biomaterials synthesis. Biomaterials, 2005, 26, 61226128.

[19] Reyderman, L.; Stavchansky, S. Electrostatic spraying and its use in drug delivery - cholesterol microspheres. Int. J. Pharm., 1995, $124,75-85$. 
[20] Hartman R.P.A. Electrohydrodynamic Atomization in the Cone. Jet Mode. From Physical Modeling to Powder Production. Ph. D. Thesis TU Delft, The Netherlands, 1998.

[21] Ciach, T. Microencapsulation of drugs by electro-hydro-dynamic atomization. Int. J. Pharm., 2006, 324, 51-55.

[22] Hayati, S.; Bailey, A. I.; Tadros, T. F. Mechanism of stable jet formation in electrohydrodynamic atomization. Nature, 1986, 319, 41-43.
[23] Hayati, S.; Bailey, A. I.; Tadros, T. F. Investigation into the mechanisms of electrohydrodynamic spraying of liquids. J. Colloid Interface Sci., 1987, 117, 205-221.

[24] Grace, J. M.; Marijnissen, J. L. M. A review of liquid atomization by electrical means. J. Aerosol Sci., 1994, 26(6), 1005-1019.

[25] Jaworek, A.; Krupa, A. Jet and drops formation in electrohydrodynamic spraying of liquids. A systematic approach. Exp. Fluids, 1999, 27, 43-52.

(C) Grafahrend et al.; Licensee Bentham Open.

This is an open access article licensed under the terms of the Creative Commons Attribution Non-Commercial License (http://creativecommons.org/licenses/by$\mathrm{nc} / 3.0 /$ ) which permits unrestricted, non-commercial use, distribution and reproduction in any medium, provided the work is properly cited. 